\title{
Embryonic Mammary Gland Development; a Domain of Fundamental Research with High Relevance for Breast Cancer Research
}

\author{
Beatrice A. Howard • Jacqueline M. Veltmaat
}

Published online: 18 May 2013

(C) Springer Science+Business Media New York 2013

Mammary gland development is often presented as a series of events happening during puberty, pregnancy, lactation and involution; even though it starts in utero, and extensive morphogenetic events occur prior to birth. During the last century, research on the embryonic phase of mammary gland development received little attention as lactation and breast cancer investigations experienced greater emphasis. Nonetheless, important contributions were made during the 1950's-1980's by Boris Balinsky, Albert Raynaud, Alain Propper, Klaus Kratochwil and Teruyo Sakakura and respective coworkers in understanding mammary development in rabbit and mouse embryos.

In part due to interests in developmental biology and birth defects per se, and in part due to the recognition of mechanistic parallels between organogenesis and carcinogenesis, the prenatal phase of mammary gland development has become more intensively studied again over the past decade. We set out to assemble this issue in such a way that it could serve as a textbook of prenatal mammary gland development, bringing together the older literature with the more recent insights. Though we succeeded in doing that for mammary development in mouse and rabbit embryos, admittedly and regrettably, this issue underrepresents insights in prenatal mammary development in monotremes, marsupials,

\footnotetext{
B. A. Howard $(\bowtie)$

Division of Breast Cancer Research, Breakthrough Breast Cancer Research Centre, The Institute of Cancer Research,

237 Fulham Road, London SW3 6JB, UK

e-mail: beatrice.howard@icr.ac.uk

J. M. Veltmaat $(\square)$

Institute of Molecular and Cell Biology,

A*STAR (Agency for Science, Technology and Research),

61 Biopolis Drive, Singapore 138673, Singapore

e-mail: jveltmaat.sc@gmail.com
}

and placentals other than mice and rabbit, including human. This is in part due to these species being less available for study, although interesting work has been and is currently being done on them. For a comprehensive review on progress in research on human breast development, we recommend a recent publication elsewhere by Gusterson and Stein [1].

We have been very fortunate in receiving the enthusiastic cooperation of many authors who have all greatly contributed to our current understanding of prenatal mammary gland development. We are particularly indebted to several esteemed Emeritus Professors, whom despite their "officially retired" status, generously dedicated their time and treasures of knowledge to this special issue. These scientists performed classical experimental embryology several decades ago and produced insights that are seminal to our current understanding of embryonic mammary development.

Among them are Alain Propper who, using rabbit embryos as a model, was the first to demonstrate that the inductive signals for mammary gland development reside in the dermis (1967), and to propose that 'wandering cells' along the mammary line contribute to growth of the mammary rudiments (1978). He joined us in co-authoring a review of morphogenesis of the embryonic mammary gland in rabbit and mouse embryos, which also serves as an introduction of developmental stages and nomenclature used in other reviews in this issue. Evolutionary biologist Olav Oftedal and developmental biologist Danielle Dhouailly teamed up especially for this occasion. Olav received the inspiration to study the evolution of the mammary gland and lactation biology from the longest and perhaps least known research efforts (1890-1922) of his lineal ancestor Alexander Graham Bell to selectively (and successfully) breed sheep for increased teat number. Danielle was a naturalist at a very young age and dedicated her career to understanding skin appendage and pattern 
formation, combining classical experimental embryology with modern molecular biology and bio-informatics. Based on her earliest experiments, she coined the concept of a continuous dialogue between the dermis and epidermis that regulates epidermal and appendage development (1977). Olav and Danielle here present their treatise with novel theories on the evolutionary biology and developmental processes contributing to the ontogeny of the mammary gland and the diversity of its phenotypes observed in different mammalian species. Between 1973 and 1991, Teruyo Sakakura established the dual origin of mesenchyme, i.e. the primary mammary mesenchyme and the fat pad precursor, in embryonic mammary gland development. She built on studies by Klaus Kratochwil establishing that these mesenchymes control the organ-specific three-dimensional pattern of branching morphogenesis of the mammary epithelium, and showed moreover that the epithelium commits to a mammary fate prior to E16 in mouse. With an interest in cancer biology, she identified developmental effects of embryonic mammary mesenchymes and the extracellular matrix component Tenascin $\mathrm{C}$ on adult normal and cancerous mammary epithelium, which made her an early proponent of a role for the stroma in mammogenesis and carcinogenesis. She revisits that idea with her co-authors in this issue. Jerry Cunha unequivocally demonstrated the epidermal origin of the mammary epithelium and the mammary epithelial cell fate promoting influence of the mammary mesenchyme (1995). His contribution to this issue is the protocol for tissue separation and recombination that he used in those studies, and may now serve to separately investigate stromal and epithelial roles of any molecule of interest.

In between their reviews is a series of reviews by scientists who started their careers when genetic manipulation had become widely available, and research activities globally shifted from tissue-interactions in organogenesis and morphogenesis to the identification of molecular regulators of such interactions. In the past decade, roles in embryonic mammary gland development have been identified for several pathways. Scientists who have contributed largely to those insights have written concise reviews of the pathway under their study. Together, they have only just begun to lift the tip of the veil regarding connections between these pathways, which remain to be fully elucidated. We have arranged the 'molecular' reviews in the sequence in which the respective molecules/signaling pathways are likely to exert their roles, although future studies may show discrepancies with this order. The series starts with a review by Jacqueline Veltmaat et coll. on $\mathrm{Hh} / \mathrm{Gli}$ signaling, followed by reviews by Pengfei Lu and HanSung Jung et coll. on FGFs; by Virginia Papaioannou et coll. on roles of Tbx 2 and Tbx 3 ; by Beatrice Howard et coll. on $\mathrm{Nrg} 3 / \mathrm{Erbb} 4$ signaling; by Paul Hamel and Kata Boras-Granic on Wnt signaling; by Marja Mikkola et coll. on Eda/TNF/NFKB signaling; and ending with a review of PTHrP signaling by Minoti Hiremath and John Wysolmerski. In the course of the past 15-20 years, John and his team have elegantly identified signaling via the PTHrP/PTHrP-R1 ligandreceptor pair as a prime example of how one signaling cascade regulates mammary development in utero and postnatally, and plays a role in breast cancer metastasis as well. Parallel roles in mammary organogenesis and tumorigenesis or metastasis are now also emerging for other pathways.

Another aspect of PTHrP signaling lies in formation of the nipple as an important accessory to the mammary gland. John Foley became interested in nipple development during his post-doctoral years in John Wysolmerski's lab, and continues this line of research independently. With members of his team, he wrote an exciting review of the evo-devo aspects of nipple formation.

Studies of embryonic mammary development should give insights relevant to human disease, including breast cancer. Similarities between mammary development and tumorigenesis in terms of molecular regulators and tissue-interactions are discussed in the 'molecular' reviews mentioned above. But positional variations in these mechanisms also exist and need to receive more attention in experimental design and documentation, as discussed in a review by Jacqueline Veltmaat, Ann Ramsdell and Esta Sterneck. The role of the mammary stroma in cancers of the mammary epithelium, as postulated 4 decades ago by Teruyo Sakakura, is reinforced in the review by Ana Soto et coll. By discussing how in utero exposure to endocrine disruptors affects the mammary mesenchyme, alters mammary gland development and creates a predisposition to breast cancer, these authors furthermore emphasize that predisposition to breast cancer can start in the womb.

Lastly, the presence and distinct developmental characteristics of stem cells in the mouse embryonic mammary glands are reviewed by Connie Eaves and Geoff Wahl and their colleagues. Of particular interest are the molecular parallels between the induction of mammogenesis, the regulation of embryonic mammary stem cell activity, and association with breast cancer with respect to e.g. FGFR and ErbB signaling, and the divergence in that respect at the level of involvement of Wnt signaling, which is required for induction and many aspects of morphogenesis, while seemingly inactive in prenatal stem cells.

To further aid those wishing to study embryonic mammary gland development, a collection of relevant techniques is appended. These are protocols for tissue separation and recombination; whole mount antibody staining; embryonic flank electroporation and culture; culture from mammary 
bud to branching stage; and bead preparation and implantation. Altogether, this issue is meant to be a resource for researchers looking for historical background, up-to-date information, and emerging new concepts in our understanding of embryonic mammary development.

We have thoroughly enjoyed putting this issue together with the enthusiastic and insightful contributions of all authors. With them, we hope this special issue facilitates and inspires others to join us in researching the prenatal stages of development of this most fascinating organ.

\section{References}

1. Gusterson BA, Stein T. Human breast development. Semin Cell Dev Biol. 2012;23(5):567-73. 